\title{
Modelling and simulation of urban traffic systems: present and future
}

\section{Feng Qiao* and Tao Liu}

Faculty of Information and Control Engineering,

Shenyang JianZhu University,

Shenyang 110168, Liaoning, China

Email: fengqiao@sjzu.edu.cn

Email: 1406221217@qq.com

*Corresponding author

\section{Haochen Sun}

School of Information Engineering,

Henan University of Science and Technology, Luoyang 471000, Henan, China

Email: hcsun1994@163.com

\section{Lingzhong Guo}

Department of Automatic Control and Systems Engineering, University of Sheffield, Sheffield, England, UK

Email:1.guo@sheffield.ac.uk

\section{Yifan Chen}

Faculty of Information and Control Engineering,

Shenyang JianZhu University,

Shenyang 110168, Liaoning, China

Email:573997389@qq.com

\begin{abstract}
The conflict of supply and demand in urban traffic system causes serious problems nowadays owing to rapid urbanisation worldwide, including traffic congestion, air pollution, etc. In this paper, the recent development of modelling and simulation of urban traffic systems is briefly overviewed, where the macro-, micro- and meso-scopic simulation models, especially the microscopic models are discussed. Three widely used microscopic traffic simulation models are analysed and the new generation traffic simulation technologies for Intelligent Transportation Systems (ITS) are discussed from the perspectives of software technology, hardware technology, and methodologies. The existing problems and challenges in modelling and simulation of urban traffic systems are evaluated and the latest trends and the potential solutions are suggested.
\end{abstract}

Keywords: modelling and simulation; urban traffic system; present and future; ITS; intelligent transportation system. 
Reference to this paper should be made as follows: Qiao, F., Liu, T., Sun, H., Guo, L. and Chen, Y. (2021) 'Modelling and simulation of urban traffic systems: present and future', Int. J. Cybernetics and Cyber-Physical Systems, Vol. 1, No. 1, pp.1-32.

Biographical notes: Feng Qiao received his BEng degree in Electrical Engineering and MSE degree in Systems Engineering from the Northeastern University, Shenyang, China in 1982 and 1987, respectively; and PhD degree in Intelligent Modelling and Control from the University of the West of England, Bristol, UK in 2005. During the period between 1987 and 2001, he worked at the Automation Research Institute of Metallurgical Industry, Beijing, China, as a Senior Engineer in Electrical and Computer Engineering. Currently, he is a Professor at the Faculty of Information and Control Engineering, Shenyang JianZhu University (SJZU), Shenyang, China. His research interests include modelling and simulation, intelligent computation, control theory and application. Currently, he is acting as the EiC of the International Journal of Simulation and Process Modelling, an EB member of the International Journal of Modelling, Identification and Control, and he serves on many international conferences as IPC and OPC members.

Tao Liu is a master's degree student of the Faculty of Information and Control Engineering, SJZU and he completed his Bachelor's degree in Automation at SJZU in July 2018. His research interests include modelling and simulation, intelligent traffic systems and optimisation.

Haochen Sun is currently a Doctoral candidate of Henan University of Science and Technology, Luoyang, China, majoring in Control Theory and Control Engineering. He received his BS degree in Electrical Engineering and Automation from Beihua University, Jilin, China and MS degree in Systems Engineering from Shenyang Jianzhu University, Shenyang, China in 2016 and 2019, respectively; and he was appointed as the General Secretary of ISSPM 2018. His research interests include modelling and simulation for complex systems, optimisation theory and application, machine learning, artificial intelligence and energy management for hybrid electric vehicles.

Lingzhong Guo obtained his $\mathrm{PhD}$ degree in Control Engineering from Bristol Robotics Laboratory (BRL), UWE in 2003. Currently, he is a Lecturer at the Department of Automatic Control and Systems Engineering, the University of Sheffield, UK. His research interest covers the modelling, identification, and simulation of complex systems, adaptive and intelligent control, and analysis and control of PDEs.

Yifan Chen is a master's degree student of the Faculty of Information and Control Engineering, SJZU and she completed her Bachelor's degree in Intelligent Systems and Automation at SJZU in July 2018. Her research interests include modelling and simulation, intelligent traffic systems and optimisation.

\section{Introduction}

With the rapid urbanisation around the world, the populations in cities are increasing exponentially along with the number of vehicles in urban areas, which makes the transportation demand increase dramatically and results in a series of traffic problems 
worldwide. In the USA, traffic congestions cost about 2.9 billion gallons of fuel and over 121 billion dollars per year (Wilkie, 2015). In Europe, pollution kills 600,000 people and the government expends more than 1,600 billion dollars every year (Mingrone et al., 2015). An efficient traffic system can reduce traffic jams, increase traffic flow, shorten travel time, and decrease air pollutants, effectively.

It has been shown that the main cause of traffic congestion is the imbalance between the increasing demand for traffic volume and the relatively insufficient supply of traffic infrastructure. To solve this conflict, many traffic system analysis techniques have been proposed over the past decades, among which traffic system simulation is one of the most important tools.

Modelling and simulation of traffic systems, as an integrated technology, has made great progress with the advancement of computer technology and programming tools since the 1950s. The traffic system simulation technology can be divided into three typical developmental stages: preliminary exploration period (1950-1970s), rapid development period (1980-1990s), and intelligent development period (since 2000). The first urban intersection simulation was completed by the British Transport \& Road Research Laboratory (TRRL) in 1951. Around 1960, traffic simulation technology was widely accepted. In 1976, SATURN, a macro-model for traffic simulation was developed (Wei et al., 2003) and the micro-traffic simulation system TRAF-NETSIM was created in 1982 (Dixon, 1997). In 2003, the Next Generation Simulation (NGSIM) Program was proposed in the USA (Alexiadis et al., 2004).

In recent years, many researchers have reviewed the modelling and simulation technology of traffic systems from different perspectives. For example, the calibration techniques and methods of macro-scopic traffic flow models were summarised by Kontorinaki et al. (2014). The recent development of the traffic and vehicle simulation tools was evaluated by Kousoulidou et al. (2013). The numerical simulation technology of traffic flow was reviewed by Mazo et al. (2015). The verification, validation and accreditation (VV\&A) of simulation models have been discussed by Tang et al. (2006). More recently, the ITS, based on Internet of Things (IoT), were reviewed by Chen et al. (2010a). In this paper, we will focus on the modelling and simulation of traffic systems in urban areas and we will provide a review about the state-of-the-art and a discussion about the challenge and potential solutions.

The remaining part of this paper is organised as follows. In Section 2, the main development of traffic system simulation technology is presented. In Section 3, the current research of macro-, micro- and meso-scopic traffic simulation models is overviewed with the emphasis on the micro-traffic simulation models and software. In Section 4, the ITS-oriented new generation traffic simulation technology is presented briefly. The problems and challenges that need to be addressed urgently are discussed in Section 5. In Section 6, future research is directed. The conclusions are drawn in Section 7.

\section{Development of traffic system simulation}

Nowadays, the study of modelling and simulation of traffic systems has attracted intensive attention in scientific and research areas. Various methods, algorithms, and strategies have been proposed and applied to solve the problems of traffic systems. The 
development of traffic simulation can approximately be divided into three stages: 19501970s, 1980-1990s, and 2000 onwards.

\subsection{0-1970s (preliminary exploration period)}

In the 1950s, researches on traffic simulation mainly focused on how to simulate the simple traffic flow. With the emergence of the first high-level programming language FORTRAN in 1951 (Backus, 1980), traffic system simulation captured the researchers' attention. In 1951, the TRRL completed the earliest intersection simulation (Ross and Gibson, 1977). Gerlough (1955) applied computer simulation technology to simulate the highway traffic. After that, many research institutions in the USA started to implement some preliminary exploratory researches on traffic system simulation, such as the simulation of intersections, car-following phenomenon, and highway ramp, etc. (Jian-wei and Zi-hua, 2003).

Around 1960, it was widely accepted that the traffic flow theory could be studied by computer simulation. Within the decade of the 1960s, many typical research works were carried out. The macro-scopic traffic simulation software NPL, the traffic control simulation software LWEIS, and the vehicle delay simulation software KELL were developed in 1962. In 1963, a simulation program AITKEN for $T$-shaped intersections and a traffic signal configuration model TRANS emerged (Katz, 1963). The green wave traffic optimisation model for arterial roads was proposed by Morgan and Little (1964), and then, based on this the MAXBAND model was developed. Gerlough and Capelle (1964), in the same year, investigated the traffic flow theory in details which laid a fair foundation for modelling and simulation of traffic systems. In 1966, the SIGOP simulation system was developed by the Federal Highway Administration (FHWA) for the signal timing optimisation (Kell and Cody, 1972). In 1967, in order to determine the optimum value of the traffic signal timing, a macro-scopic traffic simulation software TRANSYT was developed by Robertson (1969) During this period, the macro-model, with the low level of details and poor flexibility, was widely used to simulate and optimise urban traffic signals. Because of the low computer performance, it was difficult to get the expected simulation results. In spite of this, the descriptions of some traffic behaviours were proposed.

In the 1970s, the emergence of a computer language Smalltalk (Ingalls et al., 1997), the first Integrated Development Environment (IDE), has greatly driven the rapid progress of traffic simulation. With the development of computer technology, the accuracy of the traffic simulation model improved rapidly, and the functions of the simulation model were diversified. In 1971, Lieberman established a model UTCS-1 for describing the vehicular movements (Lieberman et al., 1972). In 1974, MISTRAN as a model for studying the interaction between vehicles and pedestrians at intersections was developed by the Japanese Science Police. A macro-scopic model SATURN, used for signal control at intersections, was developed by the University of Leeds in 1976 (Lu, 1999). This period also presented some simulation software to implement the multiobjective optimisation. In 1977, a macro-scopic model SOAP, jointly developed by the University of Florida and the FHWA, was able to optimise some parameters such as the signal cycle time, phase difference, and green split, etc. And the TEXAS system was developed by the University of Texas for simulating some traffic features, such as road geometry, driver's and vehicular characteristics, traffic conditions, type of intersection control, and signal timing (Jian-wei and Zi-hua, 2003). 
At this stage, traffic system simulations, limited by computer performance, were mainly implemented by large and medium-sized computers or graphics workstations. The structured program was widely used to develop the simulation software, and FORTRAN language or special simulation languages were predominant. Hence, the real traffic scenario was very difficult to be reconstructed by the simulation model, and the simulation system was non-universal, unmaintainable, non-extendable and noninteractive. So, the development of simulation software was relatively isolated and lacked a uniform standard.

\subsection{0-1990s (rapid development period)}

In the 1980s, with the rapid development of object-oriented language, traffic simulation technology was at a new stage. In this period, a hybrid macro-micro-simulation model was investigated. In 1984, a macro-micro-simulation model DYNEMO, which was successfully applied in the simulation experiment under the condition that the vehicle is being driven out of the expressway, was proposed by Schwerdtfeger. In this model, the general formula of traffic flow was used to describe the vehicle flow, and, at the same time, a single-vehicle was regarded as a unit. As computer science developed rapidly, the simulation accuracy had been greatly improved, and the micro-scopic traffic simulation models were becoming increasingly popular and were used to address a wide range of problems. A micro-scopic simulation model TRAF-NETSIM, which uses time scanning technology to simulate a single-vehicle and solves the problem of the standardisation of simulation software, was developed by the FHWA in 1982. In addition, the macro-scopic and micro-scopic simulation of the urban road network and expressway can be simulated by TRAF-NETSIM, the emergence of which meant the accuracy of the simulation and the study of traffic simulation had reached new heights (Sibley, 1985). In 1983, MISSION, which can simulate a variety of traffic conditions, was developed by Hubsehneider. At this stage, some common traffic phenomena (such as car-following, lane-changing, traffic conflict, pedestrian conflict, queue overflow etc.) and some common traffic management and control measures (such as fixed-time control, inductive control, priority control, etc.) all can be simulated.

In the $1990 \mathrm{~s}$, with the development of multimedia programming interface, the computer graphics and image processing capabilities have improved dramatically. And the appearance of Java (a general-purpose computer programming language that is concurrent, class-based and object-oriented) provided a new method for developing computer simulation software (Miller et al., 2002). Many countries began to develop or upgrade the traffic simulation software, based on the ITS. And a series of simulation software for analysing and evaluating the ITS were developed (Barceló et al., 1998). In this period, in order to meet the needs of industrial development, a high-level protocol High Level Architecture (HLA), which improved the interoperability among types of simulations and the reusability of the components of simulation models, was proposed by the USA and the other developed countries (IEEE, 2010), With the emergence of Windows, a computer operating system developed by Microsoft Corporation, visualisation, open, human-friendly interface and integration became the goals of the development of traffic simulation software. For example, in 1998, based on Windows NT/95, simulation software TSIS4.2, which has a friendly interface and integrates three simulation softwares, CORSIM, TRAFVU and ITRAF, has been developed by FHWA (Daigle et al., 1997). Some widely used traffic simulation software like PARAMICS 
(QUADSTONE, 2006), VISSIM (PTV, 2008), and AIMSUN (TSS, 2007) were developed in this period. During this period, the traffic simulation technology was improved dramatically by the progress of the modelling and simulation method, data collection, analysis and processing technology, and computing environment.

\subsection{Since 2000 (intelligent development period)}

With further study of the ITS, traffic simulation technology presents many new features, such as the higher precision and the stronger description ability of simulation models, the adaptable description of complex networks and traffic phenomena, and the collection of high-precision traffic data etc. At this stage, in order to meet the diverse needs of users, a variety of advanced application programming interfaces were provided by the commercial simulation systems. The Hardware-in-the-Loop (HIL) simulation (Bullock et al., 2004; Wells et al., 2001) and Software-in-the-Loop (SIL) simulation (Stevanovic et al., 2009), which are used in the development and test of complex real-time embedded systems, have been more popular in the field of traffic simulation. Many new intelligent control strategies and high-techs have been applied. The number of studies of online traffic simulation has increased significantly. According to the development of ITS and traffic simulation technology, this stage can be divided into two periods: 2000s and 2010s.

1 In the 2000s: In this period, traffic simulation technology had been improved, because of the development of computer technology and the emergence of new generation $\mathrm{OO}$ programming language. It was an important historical period in the process of the development of traffic simulation technology and ITS, as the Next Generation Simulation Program (NGSIM) was proposed by FHWA in 2003 (Alexiadis et al., 2004). The theories and methodologies of modelling complex systems (Gu and Chen, 2004), the simulation credibility (Ni and Leonard, 2004), the simulation tools (Cetin et al., 2002; Balmer and Raney, 2003; Champion et al., 2008; Sigel et al., 2006) and the methodologies of simulation (Wang et al., 2008; Jin and Lam, 2003) were all studied.

In terms of the theory and methods for modelling the complex systems, how to establish a formal model (Karlsson et al., 2014), which can improve the people's understanding of the objective world, is the focus. With the introduction of Agent, which is a concept in Artificial Intelligence (AI), the agent-based complex adaptive system was studied (Balaji and Srinivasan, 2010; Hsu and Liu, 2007). Some scholars also proposed that the process modelling can be implemented by the combination of Petri nets and workflow technology (Febbraro et al., 2004; Li and Li, 2010; Tzes et al., 2002). According to the development of the ITS, some innovative approaches were proposed. For example, the intelligent vehicle-infrastructure integration system could be embedded in the simulation models (Ma et al., 2009).

With the increasing complexity of the traffic simulation system, the reliability of models was paid more attention. The Verification, Validation and Accreditation (VV\&A) of models and simulations were investigated by Liu and Yang (2009); Liu et al. (2006) and Tang et al. (2006), such as the validation of simulation models based on statistical methods was studied by Kleijnen (1995), the risk-based VV\&A technology was studied by Elele and Smith (2010), and so on. With the in-depth research on the ITS, in face of the more complex system ITS, the User Program Interface (UPI), which is provided by 
commercial simulation systems, can hardly be helpful. In order to solve this problem, HIL (Engelbrecht, 2001) and SIL (Stevanovic et al., 2009) have increasingly been applied to traffic simulation.

In this period, much attention was paid to research on the interoperability and the reusability of simulation systems and the sharable function of simulation systems was regarded as a service, instead of an application. The simulation models became more modular, layered, normalised, and intelligent by using OO programming (Gowri et al., 2009), Federation Object Models (FOM), Simulation Object Models (SOM) (Kubat, 2009), and Multi-agent systems (Champion et al., 2008; Doniec and Piechowiak, 2008), etc. So, the efficiency of simulation models has been increased substantially. With the development of 3D technology and $\mathrm{OO}$ language, being networked, virtualised, intelligent, collaborative, and universal were the goals of the new generation traffic simulation technology. The multimedia visual simulation technology, which integrated the process and results of numerical simulation with some visual and auditory media, made the simulation process more intuitional and the results of simulation more comprehensible, and was able to verify the correctness of the simulation process as well. In this period, two significant simulation systems DYNAMIT (developed by MIT) (BenAkiva et al., 2001) and DYNASMART (jointly developed by the University of Texas at Austin \& the University of Maryland) (Mahmassani, 2001) and some other systems like DYNAMEQ (Mahut et al., 2003), MEZZO (Burghout et al., 2005), TRANSMODELER (Burghout et al., 2011) were all visual simulation systems, but only at the initial stage. With the increasing complexity of traffic systems, the number of computations became very expensive. To solve this problem, a collaborative distributed simulation was proposed based on Distributed Interactive Simulation (DIS) (Buss and Ruck, 2004). The virtual reality simulation technology has now widely been regarded as the most exciting and promising simulation technology. By using this technology, people can experience immersive virtual reality to visualise the real-world conditions from every angle, potential alternative control strategies, and to adjust the system parameters timely. The VR simulation has been applied to many fields (Dangelmaier et al., 2005; Volino et al., 2005; Kockro et al., 2000; Gallagher et al., 2005; Huang et al., 2001), but seldom used in the field of traffic simulation, just some preliminary studies (Ye et al., 2010), because of the huge complexity of the traffic systems.

2 In the 2010s: In this period, ITS and simulation technology had been improved dramatically, for the appearance of new technologies and the development of computer science and information technology. Traffic simulation tools for ITS were developed by the existing methods (Wang, 2010; Amditis et al., 2010) and some new technologies, such as the Internet of Things (IoT) (Guerrero-Ibanez et al., 2015), Cloud Computing (CC) (Trivedi et al., 2012), Big Data (BD) technology (Zheng et al., 2016; Faouzi et al., 2011), driverless technology (Lee et al., 2015), etc. ITS is one of the important application fields of the IoT, whose data acquisition function can strongly provide ITS with the comprehensive underlying data, the platform for publishing the traffic information, and the transport channel for transmitting data. And with the introduction of $\mathrm{CC}$ and $\mathrm{BD}$ technology, the existing data can be integrated, mined and analysed, which can contribute to establish a dynamic traffic information processing and management control platform. Thus, it is possible to process mass data efficiently and timely. 
With the further study of ITS, some new requirements for traffic simulation have been proposed. In order to adapt to the complex and diverse demands of traffic analysis of ITS, some description functions of mainstream simulation systems have been improved, such as signal control logic (Ramzanzad and Kanan, 2013), the driver's behaviour under the information environment (Lv et al., 2012), and so on. The safety and efficiency are both taken into consideration by the new generation of intelligent traffic simulation technology, and three key technologies are presented: (1) the extraction technology of the spatio-temporal traffic information, which is the supporting technology, (2) the optimisation technology of the micro-scopic traffic flow simulation model, and (3) the cooperative control technology of the multi-intersection traffic signals. In the simulation of ITS, because of the Vehicle-to-Vehicle (V2V), Vehicle-to-Pedestrian (V2P) and Vehicle-to-Infrastructure (V2I) communication, the driver's behaviour can be unfixed, so the traditional traffic simulation models need to be improved. The control strategies cannot be adapted to the real-time condition of the traffic flow by traditional control. While in the ITS, the position information of any vehicle can be obtained, then the information about traffic flow in the whole area can be acquired. By processing and analysing the acquired information, the cooperative control of multiple intersections can be implemented by the coordinated control algorithm.

Nowadays, the percentage of the implementation of ITS in the USA is up to $80 \%$ and has a relatively complete structure, which includes the Travel and Traffic Management System (TTMS), the Travel Demand Management System (TDMS), the Public Transport Operation System (PTOS), the Commercial Vehicle Operation System (CVOS), the Advanced Vehicle Control and Safety System (AVCSS), the Emergency Management System (EMS), and the Electronic Toll Collection (ETC) System. In Japan, the biggest dynamic navigation system in the world has been built, including the Public Transport Priority System (PTPS), the Traffic Information Providing System (TIPS), the Integrated Intelligent Image System (IIIS), the Advanced Driver-Assistant System (ADAS), the Pedestrian Information and Communication System (PICS), the Emergency Vehicle Priority System (EVPS), the Emergency Notification System (ENS), the Environmental Protection System (EPS), the Dynamic Guidance System (DGS), and the Vehicle Operation Management System (VOMS). In Europe, because of the geographical, historical and cultural background, the development of ITS is independently studied by each European country. Therefore, the ITS, in Europe, apart from having the same subsystems as in the USA and Japan, a wireless communication network covering the whole Europe is proposed, in order to implement some cross-regional functions, such as traffic management, navigation, ETC, etc. (Zhao et al., 2014). The ITS is also studied and applied by many other countries, such as China (Fei, 2010; Chen et al., 2013b) and South Korea (Kim et al., 2015; Lim, 2013).

In recent years, intelligent transportation technology has been evolving from the intellectualisation of a single factor to the integration of multiple factors. In the USA, the V2I cooperation system has been studied by the project IntelliDrive (Spear et al., 2010). In Europe, the Car2Car Communication Consortium (C2C-CC), which sets the standards of the V2I cooperation, has been established (Baldessari et al., 2007). And in Japan, the Advanced Safety Vehicle (ASV) (Tokuda, 2011) and the Advanced Highway System (AHS) (Kurata et al., 2011), based on V2I cooperation, have been developed. 


\section{Characteristic analysis of traffic simulation models}

According to the description precision, the traffic simulation models can be divided into three categories: macro-scopic, meso-scopic, and micro-scopic models. The micro-scopic simulation model is the most precise model, whose basic unit is a single-vehicle. In the macro-scopic model, the traffic flow can be described by the flow-density function to find the law of the motion of the whole traffic flow. The meso-scopic model, in terms of the description precision, is in between the micro-scopic and the macro-scopic models. In this section, these three kinds of the simulation models will be presented, the recent researches on the hybrid simulation model will be summarised, and at last, the microscopic simulation model, which is widely used and has the dominant advantages in describing the complex phenomena and the mechanism of the traffic systems, will be analysed in detail.

\subsection{Macro-scopic and meso-scopic simulation models}

The traffic flow is described as aggregation in macro-scopic and meso-scopic models. In macro-scopic models, the flow error function is used to determine the displacement of the vehicle. And in meso-scopic models, cells and turning flows are the basic description units, whose velocities are determined by their own velocity-density functions.

\subsubsection{The description of traffic flow by using macro-scopic models}

(a) BPR (bureau of public roads) function (Wong and Wong, 2016):

$$
T=T_{f}+T_{f} \alpha Q^{n}
$$

where $T$ is the travel time per unit of the distance; $T_{f}$ is the free-flow travel time; $Q$ is the hourly total traffic flow entering the studied region; $\alpha$ and $n$ are the model parameters.

(b) Conical volume-delay functions (Spiess, 1990):

$$
T=2+\sqrt{\alpha^{2}(1-x)^{2}+\beta^{2}}-\alpha(1-x)-\beta
$$

where $\beta$ is given as $\beta=(2 \alpha-1) /(2 \alpha-2) ; \alpha$ is any number larger than 1 ; and $x$ is given as $x=v / c, v$ being a measure of the traffic volume, and $c$ being a measure of the capacity of the road.

(c) Logit-based volume-delay functions (Wang et al., 2012):

$$
\begin{aligned}
& d=D_{l}+I_{l} \\
& D_{l}=t_{0} c_{1}\left[1 /\left[1-c_{2} /\left[1+\exp \left(c_{3}-c_{4} q / C\right)\right]\right]\right] \\
& I_{l}=d_{0} p_{1}\left[1+\left[p_{2} /\left[1+\exp \left(p_{3}-p_{4} q / X\right)\right]\right]\right]
\end{aligned}
$$

where $d$ is the total delay; $D_{l}$ and $I_{l}$ are the delay time of the vehicle on the road and in the intersection respectively; $d_{0}$ is the free-flow travel time in the intersection; $X$ is the capacity of the intersection; $t_{0}$ is the free-flow travel time on the road; $q$ is the traffic volume; and $c_{i} s$ and $p_{i} s$ are the model parameters. 
(d) Akcelic delay function (Akçelik, 1991):

$$
t=t_{0}+0.25 t_{0} r_{f}\left[z+\sqrt{z^{2}+8 J X / r_{f}}\right]
$$

where $t$ is the average travel time per unit distance; $t_{\mathrm{o}}$ is the minimum (free-flow) travel time per unit distance; $J$ is a delay parameter; $z$ is given as $z=x-1, x$ being the degree of saturation, given as $x=q / Q$, i.e. ratio of demand (arrival) flow rate to capacity; $r_{f}$ is given as $r_{f}=T_{f} / t_{0}$, i.e. ratio of flow (analysis) period to free-flow travel time.

\subsubsection{The description of traffic flow by using meso-scopic models}

In meso-scopic traffic simulation, vehicles are organised in groups called traffic cells, which consist of a list of vehicles that move together with the same traffic dynamics. And then the interactions among the cells are taken into consideration. It is an appropriate choice that the demand for simulation accuracy is not high and the scale of the road network is large. Two main descriptions of traffic flow are as follows:

a) The description of traffic flow based on traffic cells (Yang, 1997): In meso-scopic traffic simulation, two models are used to simulating the traffic stream: (1) a velocity-density model, which computes the speed for the last vehicle in the traffic cell; and (2) a cell-following model, which computes the speed of each traffic stream. And in the same cell, the speeds of the vehicles between the head and tail are interpolated. And the velocity-density model can be described by the following equation:

$$
v_{i 0}=V_{\min }+\left(V_{\max }-V_{\min }\right)\left[1-\left(K_{i} / K_{j a m}\right)^{\alpha}\right]^{\beta}
$$

where $v_{i 0}$ is the velocity of the tail vehicle of the traffic cell $i ; K_{i}$ is the density of the traffic cell $i ; K_{\text {jam }}$ is the jam density of the segment; $V_{\max }$ and $V_{\min }$ are the free-flow and the minimum speed of the segment, respectively; and $\alpha$ and $\beta$ are the model parameters, respectively.

The cell-following model can be described as follows:

$$
u_{i j}= \begin{cases}V_{\max } & d_{i j} \geq d_{\text {upper }} \\ \lambda_{j} V_{\max }+\left(1-\lambda_{j}\right) v_{j 0} & d_{i j}<d_{\text {upper }}\end{cases}
$$

where $u_{i j}$ is the cell-following speed of the first vehicle in cell $i$ travelling in direction $j$; $v_{j 0}$ is the speed of the tail vehicle in the leading cell $j ; V_{\max }$ is free-flow speed in the segment; $\lambda_{j}$ is given as $\lambda_{j}=d_{i j} / d_{\text {upper }}, d_{i j}$ being the distance from the head vehicle to the last vehicle in the leading cell, $d_{\text {upper }}$ being a predefined threshold distance.

b) The description of traffic flow based on road segmentation (Burghout, 2004): In this description, the road is divided into two parts: running part and queue part. When the vehicle drives on the road, it can be considered that the vehicle is on the running part. Then the driving speed is determined by a speed-density function, and in order 
to avoid the double counting of the vehicle delay, the "density" should be computed on the running part only. The commendatory velocity-density function is

$$
V_{v e h}= \begin{cases}V_{\text {free }} & \text { if } k<k_{\min } \\ V_{\min }+\left(V_{\text {free }}-V_{\min }\right)\left[1-\left(\frac{K-K_{\min }}{K_{\max }-K_{\min }}\right)^{\alpha}\right]^{\beta} & \text { if } k \in\left[k_{\min }, k_{\max }\right] \\ V_{\text {min }} & \text { if } k>k_{\max }\end{cases}
$$

where $V_{\text {veh }}$ is the vehicle speed; $V_{\text {free }}, V_{\min }$ are the free-flow and minimum speeds of the vehicle respectively; $K$ is the density on the running part; $K_{\min }, K_{\max }$ are the minimum and maximum density respectively; and $\alpha, \beta$ is model parameters.

\subsection{Micro-scopic simulation models}

According to the NGSIM project, the underlying models can be divided into three parts: the travel demand model, the travel behaviour model and the influential factors model (Passos et al., 2011; Park et al., 2010).

\subsubsection{Travel demand models}

In the micro-scopic traffic simulation system, the generation of traffic participants, the confirmation of the Origin-Destination (OD) matrix of all kinds of traffic participants, and the corresponding traffic distribution should be the main parts of the travel demand model.

To generate the vehicles, in micro-scopic simulation, the order and time interval of vehicles, entering the network, should be calculated by generating a random number. The acquisition of the OD matrix can be achieved by two ways: (1) inputting the OD matrix directly to the software, and (2) using the special method, provided by the software, to obtain the OD matrix, such as the OD Estimator (PARAMICS), VISUM (VISSIM), EMME/2 (AIMSUN) and so on. In terms of the description of the arrival of vehicles, two main methodologies, based on the probability theory, are presented: (1) discrete distribution, describing the countable events, and (2) continuous distribution, describing the time interval among the events. And when the vehicle is generated, the vehicle speed should be taken into consideration, for example, the vehicle travels freely on a gentle slope, the speed of the vehicle should obey the normal distribution (Liu and Ozguner, 2005).

\subsubsection{Travel behaviour models}

As an underlying model in the micro-scopic traffic simulation system, according to the driver-vehicle characteristics in the real traffic system, the travel behaviour model, which describes the state of the vehicle, during the period from entering the road network to leaving the road network, can be divided into three parts: the strategic-level model (the road network as a description unit), the tactical-level model (the road as a description unit), and the operational-level model (the lane as a description unit) (Yang and Sun, 2006). 
These three models describe the travel behaviour of the vehicle in the traffic environment, such as the path selection and the path changing are described by the strategic-level model; the lane-changing and the ramp metering are described by the tactical-level model; and the car-following and the braking are described by the operational-level model. In this paper, an example of each model will be presented respectively.

a) The path selection model - a strategic-level model: Two methods of describing the path selection are (1) setting path in a direct way and (2) selecting the path and distributing the traffic flow by given an OD matrix (Castillo et al., 2010). The first method can be used in small-scale networks, and the second method can be used in large-scale networks.

b) The lane-changing model - a tactical-level model: The lane-changing behaviour, which can be divided into two processes (judgment and enforcement), is a complex process that the driver adjusts the driving target strategy, according to the driving characteristics and the stimulation from the surroundings. In terms of these two processes, lane-changing can be classified as mandatory lane-changing (Hou et al., 2014), initiative lane-changing (Wang and $\mathrm{Li}, 2014$ ), desired lane-changing, cooperative lane-changing, oppressive lane-changing, and multi-step oppressive lane-changing (Hidas, 2005). The lane-changing models, up to now, are still studied incompletely, which include the models based on the minimum safety spacing (Jula et al., 1999), fuzzy logic (Tan, 2007), random utility theory (Zheng et al., 2004), agent (Tan, 2007), and the cellular automaton (Kukida et al. 2011) etc.

c) The car-following model - an operational-level model: The car-following model, as a basic model of micro-scopic traffic simulation, describes the action of the vehicle speed control, according to the stimulation from the surrounding traffic information. This model always is used to describe the interaction between the leading vehicle and the following vehicle, when the following vehicle cannot overtake the leading. The car-following models can be divided into three types: (1) mechanical following models, (2) psychophysical following models, and (3) compound following models.

A. Mechanical following models: This kind of models can generally be built by two methods: (1) taking the emergency brake of the leading vehicle into consideration to avoid the collision, and (2) giving an interval formula to keep a certain distance between the leading vehicle and the following vehicle. As the typical examples of this kind of models, Pipes and Forbes following models (Ni, 2016), Gazis-HermanRothery (GHR) model (Gazis et al., 1961), optimal velocity model (Bando et al., 1998), Gipps model (Vasconcelos et al., 2014) and Helly model (Li et al., 2016) are presented.

i Pipes and Forbes following models: The basic assumption of the Pipes following model is that following another vehicle at a safe distance is to allow yourself at least the length of a car between your vehicle and the vehicle ahead for every 10 miles per hour of speed at which you are travelling. The Pipes following model can be described as

$$
d_{\min }=L_{n}\left[v_{n+1}(t) / 4.47\right]+L_{n}
$$


where $d_{\min }$ is the minimum space headway between the leading and following vehicles; $L_{n}$ is the length of the leading vehicle; and $v_{n+1}(t)$ is the speed of following vehicle at time $t$.

While Forbes following model can be described as that the minimum time headway is the sum of the reaction time and the time required for the lead vehicle to traverse a distance equivalent to its length.

ii GHR model: The GHR model, also called GM model, is proposed by Gazis, Herman and Rothery, who work for General Motors. The function relation adopted in GHR model is

$$
\text { response }=f(\text { sensitivity, stimuli })
$$

and the general form can be given as the following function:

$$
a_{n+1}(t+T)=\frac{\alpha_{l, m}\left[v_{n+1}(t+T)\right]^{m}}{\left[x_{n}(t)-x_{n+1}(t)\right]^{l}}\left[v_{n}(t)-v_{n+1}(t)\right]
$$

where $x_{n}(t), v_{n}(t)$ are the position and the speed of the $n$-th vehicle at time $t$ respectively; $x_{n+1}(t), v_{n+1}(t)$ are the position and the speed of the $(n+1)$-th vehicle at time t respectively; $v_{n+1}(t+T) a_{n+1}(t+T)$ is the acceleration of the $(n+1)$-th vehicle at time $t+T ; l$ is the sensitivity denoting the spacing, i.e., $\left(x_{n}(t)-x_{n+1}(t)\right) ; m$ is the sensitivity denoting the speed $v_{n+1}(t+T) ; T$ is the time lag of response to the stimulus; and $\alpha_{l, m}$ is a constant.

iii Optimal velocity model: In optimal velocity model, the desired speed will be computed first, after the driver is stimulated by the surrounding traffic environment. This model can be presented as

$$
a_{n}(t)=c\left[v_{n}^{\text {desired }}\left(x_{n+1}(t)-x_{n}(t)\right)-v_{n}(t)\right]
$$

where $t$ is time; $x_{n}(t)$ is the positions of the $n$-th car; $x_{n+1}(t)$ is the positions of the $(n+1)$-th car, which precedes the $n$-th car; $v_{n}^{\text {desired }}\left(x_{(n+1)}(t)-x_{n}(t)\right)$ is an optimal velocity determined by the headway $x_{n+1}(t)-x_{n}(t)$, which is felt by the driver; $a_{n}(t)$ is the acceleration of the $n$-th vehicle. It is best to drive a vehicle with the optimal velocity, but in general there always exists a deviation between the optimal velocity and the real one. The deviation $\Delta v=v_{n}^{\text {desired }}\left(x_{n+1}(t)-x_{n}(t)\right)-v_{n}(t)$ is diminished by giving an acceleration $c \Delta v$ to the vehicle where the coefficient $c$ represents the sensitivity of the driver.

iv Gipps model: This model is one of the most widely used models from the collision avoidance class of models. In this model, a safe following distance is specified by two components: acceleration and deceleration sub-models, illustrated by equation (14), which outputs the velocity of each vehicle at a given time $t$ in terms of its speed at the previous step. 


$$
\left\{\begin{array}{l}
v_{n}^{a c c}(t+T)=v_{n}(t)+2.5 a_{n}^{\max } \cdot T \cdot\left[1-\frac{v_{n}(t)}{v_{n}^{\text {desired }}(t)}\right] \cdot \sqrt{0.025+\frac{v_{n}(t)}{v_{n}^{\text {desired }}(t)}} \\
v_{n}^{\text {dec }}(t+T)=-d_{n}^{\max } T+\sqrt{\left(d_{n}^{\max } T\right)^{2}+d_{n}^{\max }\left\{2\left[x_{n-1}(t)-x_{n}(t)-S_{n-1}\right]-v_{n}(t) T+\frac{v_{n-1}^{2}(t)}{d_{n-1}^{\max }}\right\}}
\end{array}\right.
$$

where $T$ is the reaction time; $v_{n}(t)$ and $v_{n-1}(t)$ are the velocities of vehicles $n$ (follower) and $n-1$ (leader) at time $t$ respectively; $v_{n}^{\text {desired }}(t)$ and $a_{n}^{\max }$ are the desired speed and the maximum acceleration of the $n$-th vehicle respectively; $d_{n}^{\max }$ and $d_{n-1}^{\max }$ are respectively the most severe braking that the $n$-th vehicle desires to undertake and the most severe braking capability of the (n-1)-th vehicle estimated by the follower; $x_{n-1}(t)$ and $x_{n}(t)$ are the longitudinal positions of the $(n-1)$-th vehicle and the $n$-th vehicle at time $t$, and $S_{n-1}$ is the effective length of the $(n-1)$-th vehicle.

v Helly model: In Helly model, the relative speed and the relative distance between the two vehicles moving on a lane are included as its main variables. The acceleration can be computed by the following equation:

$$
\left\{\begin{array}{c}
a_{n+1}(t+T)=C_{1} \Delta v(t)+C_{2}\left[\Delta x(t)-D_{n}(t+T)\right] \\
D_{n}(t+T)=\alpha+\beta v_{n+1}(t+T)+\gamma a_{n+1}(t+T)
\end{array}\right.
$$

where $D_{n}(t+T)$ is the desired following distance; $C_{i} s, \alpha, \beta, \gamma$ are the model parameters.

B. Psychophysical following models: Compared with the mechanical following models, threshold as a new conception is introduced to establish the car-following models, which are called psychophysical following models. In this kind of models, the driver's reaction, affected by the surrounding traffic conditions, is taken into consideration. Wiedemann psychophysical model (Higgs et al., 2014), Wim van Winsum psychophysical model (Winsum, 1999) and Fritzsche psychophysical model (Janson and Tapani, 2004) are all the representatives of this kind of models.

i Wiedemann psychophysical model: According to a large number of experimental investigations, the different thresholds that form the Wiedemann model are established: (1) the desired distance between the stationary vehicles $A X$, given as $X=L_{n-1}+A X a d d$ where $L_{n-1}$ is the length of the leading vehicle and $A X a d d$ is a model parameter; (2) the desired minimum following distance threshold $A B X$, which is given as $A B X=A X+B X, B X$ being given as $B X=B X$ mult $* \sqrt{v}$, where $B X m u l t$ is a model parameter and $v$ is the minimum of the speed of the following vehicle and the leading vehicle; (3) the maximum following distance $S D X$, given as $S D X=A X+E X m u l t * B X$, where Exmult is a model parameter; (4) the perception threshold $S D V$, which marks that the driver will start to react to the leading vehicle, given as $S D V=\left(\frac{\Delta x-L_{n-1}-A X}{C X}\right)^{2}$, where $C X$ is a model parameter; (5) the threshold $C L D V$, where the subject vehicle reduces the speed further to enter an 
unconscious reaction car-following episode, given as $\mathrm{CLDV}=\left(\frac{\Delta x-L_{n-1}-A X}{C L D V C X}\right)^{2}$, CLDVCS being a model parameter; (6) threshold $O P D V$, which represents the point where the driver notices the distance between his/her vehicle and the leading vehicle is increasing and should accelerate to maintain the desired space headway, given as $O P D V=C L D V^{*} O P D V m u l t$, where $O P D V m u l t$ is a model parameter.

With the thresholds above, four regimes can be defined as follows:

1) Emergency regime: When the space headway is below the $A B X$, the following vehicle is in danger and should decelerate to avoid collision. The deceleration can be obtained by equation (16):

$$
b_{n}=\frac{1}{2} \frac{(\Delta v)^{2}}{A B X-\left(\Delta x-L_{n-1}\right)}+b_{n-1}+b_{\min } \frac{A B X-\left(\Delta x-L_{n-1}\right)}{B X}
$$

where $\quad b_{\min }$ is given as $b_{\min }=B M I N a d d+B M I N m u l t * v_{n}, v_{n}$ being the speed of the following vehicle, BMINadd and BMINmult being the model parameters; $\Delta v$ is the difference of velocity; and $\Delta x$ is the front to rear distance.

2) Approaching regime: When a vehicle in the Free Driving Regime passes the Perception Threshold SDV, the vehicle will decelerate. And the deceleration can be calculated by the following:

$$
b_{n}=\frac{1}{2} \frac{(\Delta v)^{2}}{A B X-\left(\Delta x-L_{n-1}\right)}+b_{n-1}
$$

3) Following regime: The Following Regime can be divided into two sections: the Deceleration Following Regime and the Acceleration Following Regime. When a vehicle in the Approaching Regime passes the $S D V$ threshold or in the Acceleration Following Regime passes the $S D V$ threshold, the subject vehicle enters the Deceleration Following regime. And when a vehicle in the Deceleration Following Regime passes the $O P D V$ threshold or in the Emergency Regime passes the $A B X$ threshold, the subject vehicle enters the Acceleration Following regime. The acceleration of the vehicle in the Deceleration Following Regime can be calculated by the negative of equation (18).

$$
b_{\text {null }}=b_{\text {null }}
$$

where $b_{\text {null }}$ is a model parameter. And the acceleration of the vehicle in the Acceleration Following Regime can be calculated by the positive of equation (18).

4) Free driving regime: When a vehicle in the Acceleration Following Regime and crosses the $S D X$ threshold, the vehicle will enter to Free Driving Regime. In this regime, the subject vehicle will travel at the desired speed or accelerate to the desired speed, and not react to a leading vehicle. And the acceleration can be obtained by the following equations:

$$
b_{\max }=\text { BMAXmult } *\left(v_{\max }-v^{*} \text { FaktorV }\right)
$$


FaktorV $=$ FAKTORVmult

where $v_{\max }$ is the maximum speed of the vehicle; BMAXmult and FAKTORVmult are model parameters.

ii Wim van Winsum psychophysical model: By studying the driver's psychology, Wim van Winsum psychophysical model is proposed as follow:

$$
a_{n}=c \Delta T T C_{e s t}+d+\varepsilon
$$

where $a_{n}$ is the deceleration of the driver; $T T C_{\text {est }}$ is the TTC (Time-to-Collision) as estimated by the driver; $c$ and $d$ are constants; and $\varepsilon$ is a random error term.

iii Fritzsche psychophysical model: In this model, the perception thresholds, $P T N(>0)$ and $P T P(<0)$, of the speed differences can be defined as

$$
P T N=-k_{P T N}\left(\Delta x-s_{n-1}\right)^{2}-f_{x}
$$

and

$$
P T P=k_{P T P}\left(\Delta x-s_{n-1}\right)^{2}+f_{x}
$$

where $k_{P T P}, k_{P T N}$ and $f_{x}$ are the model parameters. In addition, 4 thresholds are incorporated: (1) $A D$, the desired distance between the leading and following vehicles; (2) $A R$, the risky distance, that if the distance between the leading and following vehicles is smaller than or equal to this distance, the following vehicle will decelerate heavily to avoid collisions; (3) $A S$, safe distance, that the following vehicle will accelerate, when the distance between the leading and following vehicles is greater than this distance; (4) $A B$, the braking distance, which ensures the following vehicle will not collide with the leading vehicle when the following vehicle decelerates with the maximum deceleration.

According to the thresholds above, five driving states are defined as follows:

(1) Danger: When the distance to the leading vehicle is smaller than $A R$, the following vehicle is in the danger regime, where the following vehicle will decelerate with the maximum deceleration to increase the headway.

(2) Approaching: When the speed difference is larger than $P T N$ and the space headway is between $A B, A D$ and $A R$, the following vehicle is in the approaching regime, where the following vehicle will decelerate. The deceleration can be obtained by the following expressions:

$$
\left\{\begin{array}{l}
a_{n+1}=\frac{\left(v_{n}^{2}-v_{n+1}^{2}\right)}{2 d_{c}} \\
d_{c}=x_{n}-x_{n+1}-A R+v_{n} \Delta t
\end{array}\right.
$$

where $\Delta t$ is the simulation time step and $d_{c}$ is the constraint distance.

(3) Following I: The vehicle in this regime means that the speed difference is between $P T N$ and PTP and the space headway is between $A R$ and $A D$, or the speed difference 
is larger than $P T P$ and the space headway is between $A S$ and $A R$. In this regime, the following vehicle will take no conscious actions with an assigned acceleration $b_{\text {null }}$.

(4) Following II: When the speed difference is larger than PTN and the space headway is larger than $A B$ or $A D$, the driver of the following vehicle observes that he or she is approaching to the leading vehicle, but the space headway is too long, that it is unnecessary to take any action, neither acceleration nor deceleration.

(5) Free driving: The vehicle in this regime means that the speed difference is smaller than $P T N$ and the space headway is larger than $A D$, or the absolute value of the speed difference is larger than PTP and the space headway is larger than $A S$. In this regime, the following vehicle will accelerate with a normal acceleration $a_{n}$ to achieve the desired speed. And when the desired speed is achieved, an acceleration $b_{\text {null }}$, which has some slight fluctuations, is used to maintain a quasi-constant speed.

C. Compound following models: Using mechanical following models or psychophysical following models to model the car-following behaviour, some weaknesses of these models emerge. In mechanical following models, (1) the simulation clock is not variable; (2) the perceptual field is not involved; and (3) the serial processing method is used, which does not correspond to the reality. In psychophysical following models, (1) it is very difficult to acquire the thresholds, and (2) the model parameters will vary with the environment. To make up these shortcomings, compound following models are proposed.

(i) Car-following model based on desired spacing (Hidas, 2006): This kind of models was developed specifically for the urban interrupted traffic flow conditions. A carfollowing model based on desired spacing is proposed by Peter Hidas. The model is based on the assumption that, when approaching and following the leading vehicle, the driver of the following vehicle attempts to adjust its acceleration in order to reach the desired spacing. And in this model, the desired spacing is assumed to be a linear function of the speed, different drivers have different characteristics. This model can be described by the following equation:

$$
\left\{\begin{array}{c}
x_{n}(t+T)-x_{n+1}(t+T)=\varepsilon D_{n+1}(t+T) \\
D_{n+1}(t+T)=\alpha v_{n+1}(t+T)+\beta
\end{array}\right.
$$

where $\varepsilon$ is a judgement error parameter with mean $=1.0 ; \alpha$ and $\beta$ are model parameters, which are highly dependent on the individual driver characteristics.

(ii) Car-following model based on multi-agent system (MAS)(Wu et al., 2007): An agent is an intelligent, goal-directed entity, which can accommodate new problem-solving rules incrementally, adapt and interact with surroundings, and learn and improve through interaction with the environment. So, the agent should have some features, such as autonomy, reactiveness, adaptability, communicability, and self-learning. With the systems becoming larger, the subsystems are more decentralised in temporal-spatial distribution. Then the concept of MAS is presented. An MAS, in general, has the following characteristics: high-level interactions, multiple relationships, ability to distribute data, control and resources. In the multi-agent 
based car-following model, a single-vehicle is regarded as an agent, which can receive and process the speed and acceleration of the leading vehicle and then react.

(iii) Car-following model based on fuzzy logic (González-Rojo et al., 2002): In this model, the driving behaviour is studied by the driver's future logic stage. Compared with the traditional GHR model, the fuzzy-logic model has local stability. The process of establishment of a car-following model based on fuzzy logic can be divided into the following steps: (1) determining the inputs and the outputs of the fuzzy reasoning model; (2) constructing a fuzzy control rule set and a fuzzy logic control; and (3) building reasonable fuzzy control rules. By using this model, the synchronous movement of vehicles can be realised, but masses of measurement data are needed to build sufficient fuzzy rules. And it is very difficult and complicated to calibrate the model.

iv Car-following model based on artificial neural network (ANN) (Panwai and Dia, 2007): ANN, a complex nonlinear dynamical system, which is made up of lots of connected units (also called artificial neurons) with a certain topological structure, has been applied in the traffic researches increasingly. As a new method of processing information, ANN has many satisfactory features, such as strong selflearning ability, timeliness and fault tolerance. For this kind of algorithm, some advantages are as follows: (1) determining the inputs easily, (2) realising the synchronous movement of vehicles, and (3) the driver's reaction time is not necessary to be smaller than the scanning clock. But acquiring and designing the training samples are the impediments to the application of this model.

v Car-following model based on cellular automaton (CA) (Li and Gao, 2004): The traffic system is a stochastic uncertain fuzzy large-scale nonlinear system, so it is hard to describe the traffic system accurately by using the methodology of the mathematical modelling. While the CA is an effective research tool to model such a complex system. It is because that, in the CA model, the time variable, the spatial variable and the state variable are all discrete, so the micro-scopic characteristics of the motion of particles (vehicles) can be described preferably. Thus, the CA model can be used in the study of car-following models, as the CA model can re-emerge the car-following phenomena and reveal the interactions between the vehicles. Compared with the other models, the CA model retains the complex nonlinear behaviour and other physical features and can set and label the different conditions of various simulation environments flexibly to get different simulation results.

\subsubsection{Influential factor models}

In micro-scopic traffic simulation models, influential factor models fall into 5 categories: (1) the road network, (2) the system management, (3) the operating environment, (4) the vehicle features, and (5) the traveller model.

The most commonly used models of road networks, which are described by linkconnector structure or link-node structure, abstract the traffic infrastructures in the real world. In this kind of models, two questions are taken into consideration: whether the physical features of these traffic infrastructures are described precisely; and whether the vehicle travel behaviours, influenced by the changes of the traffic infrastructures, are 
completely simulated. The influential factors of the road network generally include the type of infrastructures, the lane width, the change of slope, the auxiliary lane, etc.

The influential factors of system management can be summarised in the following categories: the provision of information and traffic control. So, the influential factors of the system management generally include the control data, the type of information, the content of information, the method for acquiring information and the type of information infrastructures (such as in-car navigation, website, broadcasting, Variable Message Sign (VMS)) etc.

As to the operating environment, events, weather and work areas are all involved. When an event occurs, the lane might be closed, or the traffic capacity might be decreased. And the changing of work areas can reduce the number of lanes, which also can make the traffic capacity decreased. Compared with the influence of events, the influence of work areas is likely to be long-term. The influence of weather involves different climates, such as fog, rain, and snow, which can have some effects on the traffic system. Additionally, the lane closure, the interaction of the adjacent vehicles, the emergency braking, the weakened capacities, the reduced speed, and the change of the lane shape are all likely to be involved.

The vehicle features usually involve the followings: type, width, length, acceleration performance, deceleration performance, speed, vehicle load, and steering performance, etc.

With the application of ITS, it is more important to do researches on traveller models. The influential factors of driver's characteristics generally include the familiar with the road network, the risk-taking in driving, the preference of the road facilities, the value of time, the compliance with traffic control (hard control such as the signal control, and soft control such as VMS).

\subsubsection{Comparative analysis of currently widely used micro-scopic traffic simulation software}

With the further development of ITS, the basic functions should be equipped in a quite complete micro-scopic traffic simulation system are as follows: (1) a strong ability to reconstruct and process the road network; (2) different types of vehicles can be generated, and the parameters of vehicles can be user-defined; (3) the conditions of vehicles can be processed in real-time, and the $\mathrm{V} 2 \mathrm{~V}$ interactions can be reflected accurately; (4) the traffic control strategy, the advanced transportation management system, and the advanced driver information system can be simulated; (5) the interface for interacting with the external applications or hardware should be provided; (6) the real-time vehicle route guidance system and the public transportation system can be simulated; and (7) the tools for analysing simulation results and the interfaces for interactive audio-visual media should be provided.

According to the above, three widely used micro-scopic simulation software PARAMICS, VISSIM and AIMSUN will be analysed and compared. In PARAMICS, the communication capability of detectors cannot be developed easily, the influences of turning velocity and vehicle turn signals are not taken into consideration, and the simulation model of the vehicle engine is not provided. In VISSIM, the influence of vehicle turn signals is not involved, the driver reaction time is immutable, and the VMS and the dynamic route guidance can hardly be implemented (Sun et al., 2019). And in AIMSUN, the subsidiary road cannot be described satisfactorily, and the influences of 
turning velocity and vehicle turn signals and the simulation model of the vehicle engine are not taken into consideration, furthermore, the influence of parking is not presented. According to the analysis, the performances of the three simulation systems are compared in Table 1. The letter grades A, B, C and D (A being the highest and D the lowest) are used to evaluate each of the performance indexes.

Table 1 The performance comparison of PARAMICS, VISSIM and AIMSUN

\begin{tabular}{lccc}
\hline Performance index & VISSIM & PARAMICS & AIMSUN \\
\hline Description model of road network & A & A & A \\
Detector and communication & A & B & A \\
Model of the vehicle unit & B & C & D \\
Signal and ramp control & A & A & A \\
Traffic management for special event & A & A & A \\
VMS & C & A & A \\
Priority of public transportation & A & A & A \\
Dynamic traffic assignment & A & A & A \\
Dynamic route guidance (DRG) & C & A & A \\
Influence model of traffic jam & A & A & A \\
Influence model of weather & A & A & A \\
Simulation of vehicle engine & A & D & D \\
\hline
\end{tabular}

\section{The new generation simulation technologies based on ITS}

With the development of high techs, in the new era, combining the high techs into one application has become a prominent feature. For ITS, in order to do the theoretical research, develop the key technology and optimise the project design, it is very important and necessary to analyse the traffic simulation results efficiently. Whether the simulation systems provide the analysis capabilities becomes the most important criterion. In recent years, some advanced ITS-oriented simulation technologies have been proposed by some mainstream traffic simulation systems, and have been widely used.

1) Multi-resolution modelling and simulation technology: For example, the microscopic description is embedded in the meso-scopic simulation model by Munoz et al. (2001). This simulation method is called integrated meso-micro-scale simulation. The study of macro-micro-scale simulation focuses on the theoretical consistency of macro- and micro-models. For example, the micro-scopic model PELOPS is aggregated into the macro-scopic model SIMONE by Lerner, and then the aggregation of micro data for macro and the disaggregation of macro data for micro are studied (Lerner et al., 2000).

2) Application programming interface technology: Under the background of ITS, many new technologies and methods are proposed. The traffic analysis presents the characteristics of the diversification, so it is very difficult to develop and implement the standardised procedure. So, the development of advanced API, provided by the 
widely-used micro-scopic traffic simulation systems, can be used to modify and expand the kernel models by users. Thus, the personalised traffic analysis demands can be satisfied.

3) Hardware-in-the-loop and software-in-the-loop technology: With the development of ITS, the control logic becomes more complex. The users can use the user-defined control logic by the application programming interface, but if the control logic is extremely complex, the simulator should be substituted by an external control module, which connects with the embedded simulation system. Accordingly, the integrated simulation can be realised. If the external control module is a real plant, the whole emulation system is called the HIL simulation system; if the external control module is software, the whole system is called the SIL simulation system.

4) Real-time simulation technology: Under the background of ITS, with the introduction of the advanced information technology, the real-time traffic simulation becomes a reality. The RTS technology, mainly used to estimate and predict the traffic situation in real time and evaluate the traffic information and the option of the management schemes, is a support tool for the real-time decision making. The mesoscopic simulation model becomes an ideal online simulation model, because of its intermediate level of detail and moderate simulation speed. In recent years, because of the better computer performance and higher simulation speed, the micro-scopic simulation model is gradually used to study the online simulation, but it is still hard to synchronise the simulations with the real world.

5) GIS-based interactive simulation technology: With the rapid development of GIS, the Network Technology (NT) and the Virtual Reality (VR) technology are used to simulate the real-world traffic conditions realistically and efficiently, and construct a high-performance virtual driving system. The digital representations of the realworld road networks are available in the form of GIS polyline road networks, which includes in online mapping and routing applications. Some simulation systems require a higher level of detail and accuracy, focusing on generating and extrapolating the subject networks from the 2-D GIS data, and recently some approaches to building 3-D geometry from the GIS data are presented (Wilkie et al., 2015).

6) Agent-based intelligent traffic simulation technology: With the further study of ITS, it is widely accepted that the ITS is a typical complex adaptive system (CAS), and it can be divided into many subsystems such as Advanced Transportation Information System (ATIS), Advanced Transportation Management System (ATMS), Advanced Driver Information System (ADIS), Advanced Vehicle Control System (AVCS) (Roger et al., 2007), Freight Management System (FMS) (Jorna et al., 2002), Electronic Toll Collection System (ETCS) (Shieh et al., 2011), Emergency Rescue System (ERS) (Martinez et al., 2010), and Advanced Public Transportation System (APTS) (US Dept. of Administration, 2006). With the introduction of the agent technology, there are two levels: (1) in the macro-scopic level, each subsystem in ITS is an agent, making all the agents collaborative to realise the information sharing, and (2) in the micro-scopic level, each vehicle, each road, each intersection and each signal is regarded as an agent. And the traffic flow, as an example of the complex systems, is emerged from vehicle agents which are mobile agents in the simulation model. And introducing the agent technology into the Intelligent 
Transportation Control System (ITCS) can make the cooperative control of Intersection-to-Intersection (I2I), vehicle-to-intersection (V2I) and vehicle-to-vehicle (V2V) a reality with many outstanding advantages such as reliability, real-time, pertinence, flexibility and expansibility.

7) The intelligent traffic simulation based on cloud computing (CC), internet of things $(I o T)$ and big data $(B D)$ : With the concept of smart city proposed and the coming of a new era of $\mathrm{CC}$, IoT and $\mathrm{BD}$, in many researches, the data generated from the traffic system and the other information systems are used to model, simulate, analyse and optimise the traffic systems. And the real traffic situation can be modelled and simulated more realistically by analysing and processing the mass data. Thus, the traffic simulation model can provide some reliable decision making for the traffic management department. And then the construction of the traffic infrastructures and the management and evaluation of road traffic system will be efficient and rational.

\section{The problems existing in the modelling and simulation of traffic systems}

For the modelling and simulation of the micro-scopic traffic systems, most of the stateof-the-art analysis and modelling are based on the micro-scopic driver behaviour, lacking the coordination and unity with macro-scopic and meso-scopic traffic characteristics. The features of humans such as dispositions, habits, and mentalities are not taken into consideration. As for the VV\&A of the simulation model, lacking credible verifications. Data samples and centralised databases, essential to calibrate the model parameters, are not considered. Under the mixed traffic conditions, the interactions between slow traffic and motor traffic are not involved in most of the current meso-scopic traffic simulation systems. And the dynamic phenomena of traffic flow can hardly be described accurately by the macro-scopic model because the dynamic characteristics of the traffic flow are oversimplified in macro-models.

Some problems in ITS modelling based on the Mobile Data Terminal (MDT) are emerging. For example, how to distinguish the different traffic modes by the data from the MDT and how to expand the data sample without distortion to acquire the OD matrix with restrictions of the communication operators and the usage rates of cellphones. For the analysis of the characteristics of the resident activities, there is no better method to model the resident activities by combining the travel information and the activity information. How to get the complete travel information from cellphone data and how to establish the corresponding relationship between the cellphone data and the user's property are still problematic without any commendable solution.

At present, GPS is widely used in APTS. By applying the GPS, the real-time bus information (such as location, time and speed) can be acquired precisely to make up the missing IC card data. And tracing the human activity and the vehicle movement become possible by using GPS and Automatic License Plate Recognition (ALPR) technology. For GPS data, the studies focus on the analysis of the macro-scopic features of the system's Spatio-temporal trajectory, while the individual travel characteristics based on GPS are seldom studied. And the lack of mining GPS big data makes the research on the real-time prediction model of human movement based on GPS exiguous. 
For ALPR technology, the macro-scopic traffic systems are mainly studied, such as discriminating the travel routes, obtaining the OD matrixes, etc. While the wireless sensor network in ALPR systems and the daily travel behaviour of every single-vehicle at the micro-scopic level are barely analysed. The problem how to analyse the mass data is solved to a certain extent by data mining, parallel computing and cloud computing, but how to use the existing technologies to find the potential movement rules of the vehicle and predict the vehicle movement is an exigent problem.

With the development of the wireless internet, the traditional Intelligent Mobile Terminals (IMT), such as smartphones, are becoming more popular, and the new types of IMT, such as the wearable computers and the IoV are emerging. The social media data, which contains a tremendous amount of traffic information, has some outstanding advantages such as large-scale samples and low cost. But there are some inherent defects in social media data: (1) the users that using the IMT daily are mainly the young and middle-aged people, so the data is incomplete and unrepresentative; (2) the data structure is not complete and the data is fragmented, so the activity location and time can be recorded accurately, but the duration cannot be acquired accurately; (3) because the individual privacy is protected, the user's private personal information can hardly be accessed, even though the information is accessed, to say the least, the integrity and facticity of the information cannot be guaranteed; and (4) the social media data are not mined and used adequately, such as the users' friends information, the text data and the image data.

The state-of-the-art micro-scopic simulation systems still cannot provide sufficient detail, and in these systems, the movement of vehicles is restricted to the centreline of the lane, a single dimension, which will not happen in the real world. And the digital representations of the cities and the geographic areas are available, but they are static, lacking dynamic elements, such as virtual people. And the full micro-scopic simulation models to the traffic data are needed to do some further researches. For example, fitting the behavioural parameters based on the data should be required, thus a larger dataset is required to avoid the simulation results being underdetermined.

\section{The future of traffic modelling and simulation}

To fit the new traffic environment and the new traffic demand, the traffic simulation model should be more deeply studied to develop the new generation traffic simulation systems: For the micro-scopic modelling and simulation, (1) the micro-macro-scale simulation should be further studied, and the micro-meso-scale traffic model should be simulated by hybrid simulation modes, such as collaborative simulation and embedded simulation; (2) the new driving behaviour, which takes the automatic driving vehicles into consideration, should be modelled with the Vehicle Automation and Communication Systems (VACS); (3) the traffic system as a typical hybrid discrete-continuous system with inference and decision, the theoretical analysis, modelling and simulation of traffic systems are still preliminary, and using the state-of-the-art research results of the discrete event simulation (DES) can contribute to the study of the modelling and simulation for traffic systems.

For the meso-scopic traffic modelling, (1) the movement rules of traffic flows (including vehicles, cycles, and pedestrians) in meso-scopic simulation should be studied; (2) the existing meso-scopic traffic models and simulation systems should be 
improved to have a better adaptability; and (3) the traffic conditions should be predicted in real-time to provide accurate data for ITS. For the macro-scopic traffic modelling, (1) the multi-lane hybrid traffic model with multi-vehicle type should be considered; (2) the initial conditions and boundary conditions of traffic flows should be solved theoretically; (3) some good features of micro-scopic traffic models can be incorporated into macroscopic traffic models, such as randomness; (4) the multi-time scale dynamic traffic flow model for the large-scale road network should be built; (5) VV\&A of simulation models based on the continuous long-term mass data should be studied; and (6) the analysis and modelling of traffic flow based on the IoV and the automatic driving vehicles should be studied.

To provide suggestions for traffic management and traffic planning, the multi-source traffic flow data should be analysed by using the new generation information technologies, such as big data, cloud computing, etc.: It is widely accepted that GPS data and licence plate data are important to understand and predict traffic conditions. There are some issues that should be more deeply studied: (1) how to get the long-term historical data directly, and how to analyse and process the mass data to find the microscopic movement characteristics of vehicles or pedestrians; (2) the micro-scopic characteristics should be reflected in the macro-scopic models to reconstruct the macroscopic laws of traffic systems and predict the macro-scopic traffic conditions.

For the social media data, there are some suggestions for further studies: (1) mining the social media data deeply to extract some users' characteristics as a supplement to the users' location information; (2) coupling with some traditional data analysis techniques to establish the correlation model between the pedestrian characteristics and the travel behaviour, and then using the social media data to estimate the pedestrian characteristics; (3) the new algorithm for deep data mining should be studied to adapt to the new devices, the new application and the more complex data; (4) for the large volume of data, the time complexity of the algorithm should be improved; (5) the new IMTs have more frequent interaction with users, and provide us many useful data, but these data have more complex format beyond the scope of the state-of-the-art researches, so how to mine and aggregate the complex data to solve the new problems should be proposed.

The driver behaviour model should be studied further with high-techs to provide the theoretical basis and the reliability assurance for the autonomous vehicle: With the development of information technology, by using a lot of state-of-the-art high-techs, the traffic information is deeply mined, and the aggregation and application of the traffic information are accomplished adequately. So, it is possible that getting the multi-source information and extracting the characteristic parameters of driving behaviour stimulated by the multi-source information. And the new generation dynamic real-time simulation technology, coupling with some advanced information technologies, such as big data and cloud computing, needs to be studied further, e.g., how to build the basic multi-resolution model based on big data and how to combine the simulation with optimisation techniques, big data analytics, and cloud computing technology. Meanwhile, the traffic congestion and the congestion transition, produced by the traffic flow guidance, should be solved timely and effectively, otherwise, it will bring forth negative impacts on the application of urban traffic network and ITS. And the development of the driverless technology and pan-information transportation, it is foreseeable that in the future the automatic unmanned driving technology will be the core of all the transportation and the transportation system will be fully automatic and traffic-light-free. If it arrives, the sensor-laden vehicles will pass through the intersections by communicating with each 
other, rather than grinding to a halt at the traffic signals. Accordingly, the transportation system will be zero-accident, zero-casualty, zero-congestion and zero-emission.

The new generation agent-oriented programming should be developed, as well as the intelligent modelling and simulation technology: For the driver behaviour in carfollowing situations, the variables should be dynamic, multi-rule, and perceptive. And the researchers should note the interdisciplinary studies, e.g., the human factors should be given more consideration, and the study of driver's behaviour and driver's psychophysiological characteristics should be combined with the cognitive psychology, the brain science, the physiology, the biology, and the praxeology. And some variables of consciousness or unconsciousness and some traffic-related parameters can be used to improve the performance of the simulation model of driving behaviour. The dynamic scheduling and real-time programming can be adopted to improve the adaptivity and the self-correcting capability of the simulation model of driving behaviour. By using the deep convolutional neural network and deep reinforcement learning, the vehicles will closely resemble human reasoning, i.e., the vehicles can make decisions independently in extreme environments and emergency situations.

The intermodal passenger transport hub (IPTH) should be provided by the urban and peri-urban traffic simulation system: A transport hub is a place where passengers are exchanged between vehicles or between public transport modes. And the construction of transport hubs is considerably behind the growth of traffic demands, and the function and structure of the hub are designed badly and unpopular. These problems all make the operating efficiency of the urban transport system extremely low. So, with the city scale becoming larger and the transport modes becoming more diversified, the simulation system for the passenger transport hub should include all the major public transport, e.g., train stations, rapid transit stations, bicycle parking stations, bus stations, taxi stations, tram stops, airports and ferry slips. And, meanwhile, it is necessary to complement and improve the theory and methodology for modelling, simulating, evaluating, and managing the intermodal passenger transport hub, and it is required that a perfect comprehensive evaluation system for the passenger transport hub should be developed. Thus, the evaluation system can provide a better plan for the traffic department to organise and optimise the traffic system so as to improve the traffic capacity.

\section{Conclusions}

In this paper, the historical development of modelling and simulation of traffic systems has been reviewed briefly, and categorised into three stages. The widely used traffic simulation models are summarised, among which special attention has been paid to the third stage with respect to ITS due to its importance. Furthermore, the current researches on macro-, micro- and meso-scopic traffic simulation models are overviewed. More specifically, three widely used micro-scopic simulation software PARAMICS, VISSIM, and AIMSUN have been compared to assist researchers in selecting the proper tool to finish their specific simulation study. Then the key features and the development status of the new generation traffic simulation technologies based on ITS are presented. So far, traffic modelling and simulation have evolved to be an imperative tool for highperformance traffic design and management systems. From our comparative analysis of currently widely used micro-scopic traffic simulation software, it can be concluded that to each of them has their own pros and cons although the performance with respect to 
some specific aspects can be excellent. The future development of traffic modelling and simulation may lie in the hybrid combination of these existing methods, enhanced by advanced theory, technology, and methods including big data, machine learning, artificial intelligence, and quantum computing.

\section{References}

Akçelik, R. (1991) 'Travel time functions for transport planning purposes: Davidson's function, its time-dependent form and an alternative travel time function', Australian Road Research, Vol. 21, No. 3, pp.1-15.

Alexiadis, V., Colyar, J., Halkias, J., Hranac, R. and Mchale, G. (2004) 'The next generation simulation program', ITE Journal, Vol. 74, No. 8, pp.22-26.

Alexiadis, V., Colyar, J., Halkias, J., Hranac, R. and Mchale, G. (2004) 'The next generation simulation program', ITE Journal, Vol. 74, No. 8, pp.22-26.

Amditis, A., Bimpas, M., Thomaidis, G., Tsogas, M., Netto, M., Zipser, S., Etemad, A., Lio, M.D. and Cicilloni, R. (2010) 'A situation-adaptive lane-keeping support system: overview of the safelane approach', IEEE Transactions on Intelligent Transportation Systems, Vol. 11, No. 3, pp.617-629.

Backus, J. (1980) 'Programming in America in the 1950s - some personal impressions', A History of Computing in the Twentieth Century, pp.125-135.

Balaji, P.G. and Srinivasan, D. (2010) 'Multi-agent system in urban traffic signal control', IEEE Computational Intelligence Magazine, Vol. 5, No. 4, pp.43-51.

Balakrishna, R., Morgan, D., Slavin, H. and Yang, Q. (2011) 'Large-scale simulation tools for transportation planning and traffic operations management', International IEEE Conference on Intelligent Transportation Systems, IEEE, Vol. 110, pp.230-235.

Baldessari, R., Bödekker, B., Deegener, M., Festag, A., Franz, W., Christopher, K.C., Kosch, T., Kovacs, A., Lenardi, M., Menig, C., Peichl, T., Röckl, M., Seeberger, D., Straßberger, M., Stratil, H., Vögel, H.J., Weyl, B. and Zhang, W. (2007) 'Car-2-car communication consortium - manifesto', IEEE Vehicular Technology Conference, pp.1-95.

Balmer, M. and Raney, B. (2003) 'Agent-based activities planning for an iterative traffic simulation of Switzerland - activity time allocation', British Journal of Haematology, Vol. 89, No. 2, pp.380-385.

Bando, M., Hasebe, K., Nakanishi, K. and Nakayama, A. (1998) 'Analysis of optimal velocity model with explicit delay', Physical Review E Statistical Physics Plasmas Fluids \& Related Interdisciplinary Topics, Vol. 58, No. 5, pp.5429-5435.

Barceló, J., Bernauer, E., Breheret, L., Canepari, G., Taranto, C.D., Ferrer, J., Fox, K., Gabard, J., Liu, R. (1998) Simulation Modelling Applied to Road Transport European Scheme Tests (SMARTEST) - Review of MicroSimulation Models. Available online at: http://citeseerx.ist.psu.edu/viewdoc/summary?doi=10.1.1.132.3569

Ben-Akiva, M., Bierlaire, M., Burton, D., Koutsopoulos, H.N. and Mishalani, R. (2001) 'Network state estimation and prediction for real-time traffic management', Networks and Spatial Economics, Vol. 1, Nos. 3/4, pp.293-318.

Bullock, D., Johnson, B., Wells, R.B., Kyte, M. and Li, Z. (2004) 'Hardware-in-the-loop simulation', Transportation Research Part C, Vol. 12, No. 1, pp.73-89.

Burghout, W. (2004) 'Hybrid microscopic-mesoscopic traffic simulation', Computer Engineering, pp.1-186.

Burghout, W., Koutsopoulos, H. and Andréasson, I. (2005) 'Hybrid mesoscopic-microscopic traffic simulation', Transportation Research Record Journal of the Transportation Research Board, No. 1, pp.218-255.

Buss, A. and Ruck, J. (2004) 'Joint modeling and analysis using XMSF web services', Conference on Winter Simulation, Vol. 1, pp.1032-1038. 
Castillo, E., Gallego, I. and Rivas, A. (2010) 'Matrix tools for general observability analysis in traffic networks', IEEE Transactions on Intelligent Transportation Systems, Vol. 11, No. 4, pp.799-813.

Cetin, N., Kai, N., Raney, B. and Voellmy, A. (2002) 'Large-scale multi-agent transportation simulations', Computer Physics Communications, Vol. 147, Nos. 1/2, pp.559-564.

Champion, A., Auberlet, J.M. and Kolski, C. (2008) 'Behaviour based on decision matrices for a coordination between agents in a urban traffic simulation', Applied Intelligence, Vol. 28, No. 2, pp.121-138.

Champion, A., Auberlet, J.M. and Kolski, C. (2008) 'Behaviour based on decision matrices for a coordination between agents in an urban traffic simulation', Applied Intelligence, Vol. 28, No. 2, pp.121-138.

Chen, L., Li, Z. and Jiang, S. (2013a) 'An overview of intelligent transportation systems based on the internet of things', Intelligent Computer and Applications, Vol. 3, No. 5, pp.43-45.

Chen, X., Zhou, G., Yang, Y. and Huang, H. (2013b) 'A newly developed safety-critical computer system for china metro', IEEE Transactions on Intelligent Transportation Systems, Vol. 14, No. 2, pp.709-719.

Daigle, G., Krueger, G.D., Clark, J. (1997) 'TSIS: advanced traffic software tools for the user', Traffic Congestion and Traffic Safety in the 21st Century: Challenges, Innovations, and Opportunities, pp.563-569.

Dangelmaier, W., Fischer, M., Gausemeier, J., Grafe, M. and Matysczok, C. et al. (2005) 'Virtual and augmented reality support for discrete manufacturing system simulation', Computers in Industry, Vol. 56, No. 4, pp.371-383.

Dixon, M. (1997) 'Calibrating and validating TRAF-NETSIM model of single-point urban interchange', Transportation Research Record, Vol. 1591, No. 1, pp.38-44.

Doniec, A. and Piechowiak, S. (2008) 'A behavioral multi-agent model for road traffic simulation', Engineering Applications of Artificial Intelligence, Vol. 21, No. 8, pp.1443-1454.

Elele, J.N. and Smith, J. (2010) 'Risk-based verification, validation, and accreditation process', Proceedings of SPIE - The International Society for Optical Engineering, pp.77050E-77050E-11. Doi: 10.1117/12.852419.

Engelbrecht, R. (2001) 'Using hardware-in-the-loop traffic simulation to evaluate traffic signal controller features', Proceedings of the Conference of the IEEE Industrial Electronics Society (IECON'01), Vol. 3, pp.1920-1925.

Faouzi, N.E.E., Leung, H. and Kurian, A. (2011) 'Data fusion in intelligent transportation systems: progress and challenges - a survey', Information Fusion, Vol. 12, No. 1, pp.4-10.

Febbraro, A.D., Giglio, D. and Sacco, N. (2004) 'Urban traffic control structure based on hybrid petri nets', IEEE Transactions on Intelligent Transportation Systems, Vol. 5, No. 4, pp.224-237.

Fei, Z. (2010) 'The current situation and development thinking of the intelligent transportation system in China', International Conference on Mechanic Automation and Control Engineering, IEEE, pp.2826-2829.

Gallagher, A.G., Ritter, M., Champion, H., Higgins, G., Fried, M.P. and Moses, G. (2005) 'Virtual reality simulation for the operating room', Annals of Surgery, Vol. 241, No. 2, pp.364-372.

Gazis, D.C., Herman, R. and Rothery, R.W. (1961) 'Nonlinear follow-the-leader models of traffic flow', Operations Research, Vol. 9, No. 4, pp.545-567.

Gerlough, D.L. (1955) Simulation of Freeway Traffic on a General-Purpose Discrete Variable Computer, PhD Dissertation, UCLA.

Gerlough, D.L., Capelle, D.G. (1964) An Introduction to Traffic Flow Theory, Highway Research Board (U.S.).

González-Rojo, S., Slama, J.G., Lopes Pereira, A. and Mora-Camino, F. (2002) 'A fuzzy logic approach for car-following modelling', Systems Analysis Modelling Simulation, Vol. 42, No. 5, pp.735-755. 
Gowri, A., Venkatesan, K. and Sivanandan, R. (2009) 'Object-oriented methodology for intersection simulation model under heterogeneous traffic conditions', Advances in Engineering Software, Vol. 40, No. 10, pp.1000-1010.

$\mathrm{Gu}$, S.S. and Chen, Y. (2004) 'Modeling and simulation of complex adaptive system - a traffic simulation system', Complex Systems and Complexity Science, Vol. 1, No. 1, pp.82-88 (in Chinese).

Guerrero-Ibanez, J.A., Zeadally, S. and Contreras-Castillo, J. (2015) 'Integration challenges of intelligent transportation systems with connected vehicle, cloud computing, and internet of things technologies', IEEE Wireless Communications, Vol. 22, No. 6, pp.122-128.

Hidas, P. (2005) 'Modelling vehicle interactions in microscopic simulation of merging and weaving', Transportation Research Part C, Vol. 13, No. 1, pp.37-62.

Hidas, P. (2006) 'Evaluation and further development of car following models in microscopic traffic simulation', Urban Transport XII Urban Transport \& the Environment in Century, pp.287-296.

Higgs, B., Abbas, M. and Medina, A. (2011) 'Analysis of the Wiedemann car following model over different speeds using naturalistic data', Proceedings of the $3 \mathrm{rd}$ International Conference on Road Safety and Simulation, Indiana, USA, pp.1-22.

Hou, Y., Edara, P. and Sun, C. (2014) 'Modeling mandatory lane changing using Bayes classifier and decision trees', IEEE Transactions on Intelligent Transportation Systems, Vol. 15, No. 2, pp.647-655.

Hsu, P.L. and Liu, H.H. (2007) 'The multi-agent system with an adaptive-fuzzy algorithm for flow control of traffic networks', IEEE International Conference on Systems, Man and Cybernetics, IEEE, Vol.17, pp.3300-3304.

Huang, B., Jiang, B. and Li, H. (2001) 'An integration of GIS, virtual reality and the Internet for visualization, analysis and exploration of spatial data', International Journal of Geographical Information Science, Vol. 15, No. 5, pp.439-456.

IEEE, B.E. (2010) 'IEEE standard for modeling and simulation (M\&S) high level architecture (HLA) - framework and rules', C/SI - Simulation Interoperability Stds Organization/Stds Activity Committee, IEEE, Computer Society, IEEE, pp.1-378.

Ingalls, D., Kaehler, T., Maloney, J., Wallace, S. and Kay, A. (1997) 'Back to the future: the story of Squeak, a practical smalltalk written in itself', ACM SIGPLAN Notice, Vol. 32, pp.318-326.

Janson, J. and Tapani, O.A. (2004) 'Comparison of car-following models', Transportation Research Record Journal of the Transportation Research Board, No. 1, pp.116-127.

Jian-wei, H. and Zi-hua, Z. (2003) 'Application analysis of traffic simulation technology', International Conference on Urban Transport.

Jin, M. and Lam, S.H. (2003) 'A virtual-reality based integrated driving-traffic simulation system to study the impacts of intelligent transportation systems (ITS)', International Conference on Cyberworlds, IEEE Computer Society, IEEE, Singapore.

Jorna, R., Van Drunen, E. and Anderson, S.M. (2002) 'Integrating traffic management systems with freight transport management systems', Biophysical Journal, Vol. 108, No. 2, pp.627a-628a.

Jula, H., Kosmatopoulos, E.B. and Ioannou, P.A. (1999) 'Collision avoidance analysis for lane changing and merging', IEEE Transactions on Vehicular Technology, Vol. 49, No. 6, pp.2295-2308.

Karlsson, D., Eles, P. and Peng, Z. (2014) 'Model validation for embedded systems using formal method-aided simulation', IET Computers and Digital Techniques, Vol. 2, No. 6, pp.413-433.

Katz, J.H. (1963) 'Simulation of a traffic network', Communications of the ACM, Vol. 6, No. 6, pp.480-486.

Kell, J.H. and Cody, R.B. (1972) 'Methods for network optimization: SIGOP', Proceedings of the Institute of Traffic Engineers, pp.190-193. 
Kim, J., Moon, Y.J. and Suh, I.S. (2015) 'Smart mobility strategy in Korea on sustainability, safety and efficiency toward 2025', IEEE Intelligent Transportation Systems Magazine, Vol. 7, No. 4, pp.58-67.

Kleijnen, J.P.C. (1995) 'Statistical validation of simulation models', European Journal of Operational Research, Vol. 87, No. 1, pp.21-34.

Kockro, R. A., Serra, L., Tseng-Tsai, Y., Chan, C., Yih-Yian, S., Gim-Guan, C., Lee, E., Hoe, L. Y., Hern, N. and Nowinski, W. L. (2000) 'Planning and simulation of neurosurgery in a virtual reality environment', Neurosurgery, Vol. 46, No. 1, pp.118-135.

Kontorinaki, M., Spiliopoulou, A., Papamichail, I., Papageorgiou, M., Tyrinopoulos, Y. and Chrysoulakis, J. (2014) 'Overview of nonlinear programming methods suitable for calibration of traffic flow models', Operational Research, Vol. 15, No. 3, pp.327-336.

Kousoulidou, M., Fontaras, G. and Lonza, L. (2013) 'Overview of emission and traffic models and evaluation of vehicle simulation tools', Journal of Anhui Technical College of Water Resources and Hydroelectric Power, Vol. 27, No. 4, pp.237-244.

Kubat, C. (2009) Scenario based Distributed Manufacturing Simulation using HLA Technologies, Elsevier Science Inc.

Kukida, S., Tanimoto, J. and Hagishima, A. (2011) 'Analysis of the influence of lane changing on traffic-flow dynamics based on the cellular automaton model', International Journal of Modern Physics C, Vol. 22, No. 03, pp.271-281.

Kurata, R., Aikawa, T. and Kamogashira, D. (2011) 'Realization of the AHS service based on the vector image processing method', Proceedings of the 18th ITS World Congress, Orlando Florida, USA.

Lee, J.G., Kim, K.J., Lee, S. and Shin, D.H. (2015) 'Can autonomous vehicles be safe and trustworthy? Effects of appearance and autonomy of unmanned driving systems', International Journal of Human-Computer Interaction, Vol. 31, No. 10, pp.682-691.

Lerner, G., Hochstaedter, A., Kates, R., Demir, C., Meier, J. and Poschinger, A. (2000) 'The interplay of multiple scales in traffic flow: coupling of microscopic, mesoscopic, and macroscopic simulation', Proceedings of The 7th World Congress on Intelligent Systems, Turin, Italy.

Li, J. and Li, Q. (2010) 'Modeling of urban traffic system based on dynamic stochastic fluid petri net', Journal of Transportation Systems Engineering and Information Technology, Vol. 10, No. 1, pp.485-491.

Li, K. and Gao, Z. (2004) 'Cellular automation model of traffic flow based on the car-following model', Chin. Phys. Lett., Vol. 21, No. 11, pp.2120-2123.

Li, Q., Qiao, F. and Yu, L. (2016) 'Calibration of car-following models considering the impacts of warning messages from tablet/smartphone application', Journal of Transportation Technologies, Vol. 6, No. 2, pp.61-75.

Lieberman, E.B., Worrall, R.D. and Bruggeman, J.M. (1972) 'Logical design and demonstration of UTCS-1 network simulation model', TRIS and ITRD Database, pp.46-56.

Lim, S. (2013) 'Standardization of intelligent transportation system in Korea', Biochemistry, Vol. 24, No. 24, pp.713-719.

Liu, F. and Yang, M. (2009) 'An optimal design method for simulation verification, validation and accreditation schemes', Society for Computer Simulation International. Doi: $10.1177 / 0037549709103652$.

Liu, X.T., Liu, L. and Sun, W. (2006) 'Simulation system VV\&A and its standard/principle research', Computer Simulation, Vol. 23, No. 3, pp.61-66.

Liu, Y. and Ozguner, U. (2005) 'Quantitative evaluation of inter-vehicle communication effect in highway transportation from micro and macro views', International Journal of Vehicle Information and Communication Systems, Vol. 1, Nos. 1/2, pp.152-180.

Lu, H.P. (1999) Urban Traffic Management Modernization (in Chinese), China Communication Press. 
Lv, M., Hou, W. and Tian, X. (2012) 'The method of virtual driving behavior in active safety based on information fusion', Advances in Information Sciences and Service Sciences, Vol. 4, No. 11, pp.337-343.

Ma, Y., Chowdhury, M., Sadek, A. and Jeihani, M. (2009) 'Real-time highway traffic condition assessment framework using vehicle - infrastructure integration (vii) with artificial intelligence (ai)', IEEE Transactions on Intelligent Transportation Systems, Vol. 10, No. 4, pp.615-627.

Mahmassani, H.S. (2001) 'Dynamic network traffic assignment and simulation methodology for advanced system management applications', Networks and Spatial Economics, Vol. 1, Nos. 3/4, pp.267-292.

Mahut, M., Florian, M. and Tremblay, N. (2003) 'Space-time queues and dynamic traffic assignment: a model, algorithm and applications', Asian Journal of Psychiatry, Vol. 12, No. 12 , pp.1-2.

Martinez, F.J., Toh, C.K., Cano, J.C., Calafate, C.T. and Manzoni, P. (2010) 'Emergency services in future intelligent transportation systems based on vehicular communication networks', IEEE Intelligent Transportation Systems Magazine, Vol. 2, No. 2, pp.6-20.

Mazo, M.J.M., Valencia, J. and Tost, G.O. (2015) 'Numerical simulation analysis of a traffic model', Analysis, Modelling, Optimization, and Numerical Techniques, Springer International Publishing, pp.363-371.

Miller, J.A., Nair, R.S., Zhang, Z. and Zhao, H. (2002) 'JSIM: a Java-based simulation and animation environment', Proceedings of the SCS Simulation Symposium, IEEE, USA, pp.31-42.

Mingrone, L., Pignataro, G. and Roscia, M. (2015) 'Smart urban electric transport system: an innovative real model', International Conference on Renewable Energy Research and Applications, IEEE, pp.1457-1462.

Morgan, J.T. and Little, J.D.C. (1964) 'Synchronizing traffic signals for maximal bandwidth', Operations Research, Vol. 12, No. 6, pp.896-912.

Munoz, L., Gomes, G., Yi, J. and Toy, C. (2001) 'Integrated meso-microscale traffic simulation of hierarchical AHS control architectures', Proceedings of the Intelligent Transportation Systems, IEEE, pp.82-87.

Ni, D. (2016) 'Chapter 13 - pipes and forbes models. Traffic Flow Theory, pp.185-196.

Ni, D. and Leonard, J.D. (2004) 'Systematic approach for validating traffic simulation models', Transportation Research Record: Journal of the Transportation Research Board, pp.20-31.

Panwai, S. and Dia, H. (2007) 'Neural agent car-following models', IEEE Transactions on Intelligent Transportation Systems, Vol. 8, No. 1, pp.60-70.

Park, B., Van Arem, B. and Van Noort, M. (2010) 'Directions for next generation microscopic traffic simulation modeling tool under the IntelliDrive environment', British Journal of Clinical Pharmacology, Vol. 68, No. 2, pp.169-181.

Passos, L.S., Rossetti, R.J.F. and Kokkinogenis, Z. (2011) 'Towards the next-generation traffic simulation tools: a first appraisal', Information Systems and Technologies, pp.1-6.

PTV - Planung transport Verkehr AG. (2008) VISSIM 5.0 User Manual, PTV, Carlsruhe, Germany.

QUADSTONE (2006) PARAMICS 5.0 User Manual, Quadstone, Edinburgh, UK.

Ramzanzad, M. and Kanan, H.R. (2013) 'A new method for design and implementation of intelligent traffic control system based on fuzzy logic using FPGA', Fuzzy Systems, IEEE, pp.1-4.

Robertson, D.I. (1969) 'TRANSYT method for area traffic control', Traffic Engineering and Control, Vol. 10, No. 6, pp.181-182.

Roger, S. (2007) 'ITS connections: saving time, saving lives: proceedings: 15 th world congress on intelligent transportation systems and ITS America's 2008 annual meeting, 16-20 November, New York, New York, USA', Journal of Non-Crystalline Solids, Vol. 353, No. 47, pp.4570-4574. 
Ross, P. and Gibson, D. (1977) 'Review of road traffic network simulation models', 56th Annual Meeting of the Transportation Research Board, Washington DC, 24-28 January, pp.36-41.

Shieh, W.Y., Hsu, C.C., Tung, S.L., Lu, P.W., Wang, T.H. and Chang, S.L. (2011) 'Design of infrared electronic-toll-collection systems with extended communication areas and performance of data transmission', IEEE Transactions on Intelligent Transportation Systems, Vol. 12, No. 1, pp.25-35.

Sibley, S.W. (1985) 'NETSIM for microcomputers', Operations and Traffic Management, Vol. 49, pp.54-59.

Sigel, A., Douglas, J.A. and Eghbali-Webb, M. (2006) 'Use of hardware-in-the-loop traffic simulation in a virtual environment', International Conference on Applications of Advanced Technology in Transportation, Vol. 141, pp.635-640.

Spear, B., Vandervalk, A. and Snyder, D. (2010) Roadway Geometry and Inventory Trade Study for IntelliDriveSM Applications, Report, Information Technology.

Spiess, H. (1990) 'Conical volume-delay functions: technical note', Transportation Science, Vol. 24, No. 2, pp.153-158.

Stevanovic, A., Abdelrahim, A., Zlatkovic, M. and Amin, E. (2009) 'Microscopic modeling of traffic signal operations: comparative evaluation of hardware-in-the-loop and software-in-theloop simulations', Transportation Research Record: Journal of the Transportation Research Board, pp.143-151.

Stevanovic, A., Stevanovic, J. and Martin, P.T. (2009) 'Optimizing signal timings from the field: VISGAOST and VISSIM-ASC/3 software-in-the-loop simulation', Transportation Research Record, Vol. 45, pp.114-120.

Sun, H., Qiao, F. and Wang, Z. (2019) 'A two-level identification model for selecting the coordination strategy for the urban arterial road based on fuzzy logic', International Journal of Simulation and Process Modelling, Vol. 14, No. 6, pp.478-487.

Tan, M.C. (2007) 'Simulation model of vehicle lane-changing based on agent and fuzzy logic', Journal of Systems Engineering, Vol. 22, No. 1, pp.40-45.

Tang, J.B., Zha, Y.B. and, Li, G. (2006) 'An overview of the research on VV\&A in simulation', Computer Simulation, Vol. 23, No. 11, pp.82-85. (in Chinese)

Tokuda, K. (2011) ITS wireless Communications Systems in EV Generation, Technical Report of IEICE RCS, 111.

Trivedi, P., Deshmukh, K. and Shrivastava, M. (2012) 'Cloud computing for intelligent transportation system', International Journal of Soft Computing and Engineering, Vol. 2, No. 3.

TSS - Transport Simulation Systems (2007) AIMSUN 6.0 User Manual, TSS, Barcelona, Spain.

Tzes, A., Kim, S. and Mcshane, W. (2002) 'Applications of petri networks to transportation network modeling', IEEE Transactions on Vehicular Technology, Vol. 45, No. 2, pp.391-400.

US Dept. of Administration (2006) Advanced Public Transportation Systems: The State of the Art Update 2006, US Dept. of Administration.

Vasconcelos, L., Neto, L., Santos, S., Silva, A.B. and Seco, A. (2014) 'Calibration of the gipps carfollowing model using trajectory data $\Sigma^{2}$ ', Transportation Research Procedia, Vol. 3, No. 9, pp.952-961.

Volino, P., Cordier, F. and Magnenat-Thalmann, N. (2005) 'From early virtual garment simulation to interactive fashion design', Computer-Aided Design, Vol. 37, No. 6, pp.593-608.

Wang, C., Chen, G., Liu, Y. and Horne, M. (2008) 'Virtual-reality based integrated traffic simulation for urban planning', International Conference on Computer Science and Software Engineering, IEEE Computer Society, Vol. 2, pp.1137-1140.

Wang, F.Y. (2010) 'Parallel control and management for intelligent transportation systems: concepts, architectures, and applications', IEEE Transactions on Intelligent Transportation Systems, Vol. 11, No. 3, pp.630-638. 
Wang, Q. and Li, Z.L.L. (2014) 'Investigation of discretionary lane-change characteristics using next-generation simulation data sets', Journal of Intelligent Transportation Systems, Vol. 18, No. 3, pp.246-253.

Wang, W., Jing, M., Liu, D. and Wang, J. (2012) 'Study on capacity limitation-multipath routing traffic allocation based on LOGIT model and BPR impedance function', Journal of Highway and Transportation Research \& Development, Vol. 29, No. S1, pp.81-85. (in Chinese)

Wei, M., Yang, F.T. and Cao, Z.Q. (2003) 'A review of development and study on the traffic simulation’, Acta Simulata Systematica Sinica, Vol. 15, No. 8, pp.1179-1097.

Wells, R.B., Fisher, J., Zhou, Y. and Johnson, B.K. (2001) 'Hardware and software considerations for implementing hardware-in-the loop traffic simulation', Proceedings of the Conference of the IEEE Industrial Electronics Society (IECON'01), IEEE, Vol. 1, pp.1915-1919.

Wilkie, D. (2015) Simulating, Reconstructing, and Routing Metropolitan-Scale Traffic, Dissertations \& Theses - Gradworks, Vol. 21, No. 37.

Wilkie, D., Sewall, J., Li, W. and Lin, M.C. (2015) 'Virtualized traffic at metropolitan scales', Frontiers in Robotics and Ai, Vol. 2, No. 11, pp.1-10. Doi: 10.3389/frobt.2015.00011.

Winsum, W.V. (1999) 'The human element in car following models', Transportation Research Part F Traffic Psychology and Behaviour, Vol. 2, No. 4, pp.207-211.

Wong, W. and Wong, S.C. (2016) 'Network topological effects on the macroscopic bureau of public roads function', Transportmetrica A Transport Science, Vol. 12, No. 3, pp.272-296.

$\mathrm{Wu}, \mathrm{C}$., Yan, X. and Ma, X. (2007) 'A new car-following model based on multi-agent system', International Symposium on Distributed Computing and Applications to Business, Engineering and Science, Vol. II, pp.137-140.

Yang, Q. (1997) 'A simulation laboratory for evaluation of dynamic traffic management system', Simulation, pp.1-193.

Yang, X.G. and Sun, J. (2006) 'Microscopic traffic simulation and experimental system under ITS', Journal of Changsha University of Science and Technology, Vol. 3, No. 3, pp.43-48. (in Chinese)

Ye, X.L., Yang, Y.H. and Zou, L.P. (2010) 'Research on the application of virtual reality technology in transportation', International Conference on Mechanic Automation and Control Engineering, IEEE, pp.2887-2890.

Zhao, N., Yuan, J.B. and Han, X.U. (2014) 'Survey on intelligent transportation system', Computer Science, Vol. 28, No. 1, pp.1-3.

Zheng, H., Rong, J. and Ren, F.T. (2004) 'A lane changing model based on random utility theory', Journal of Highway and Transportation Research and Development, Vol. 21, No. 5, pp.88-91. (in Chinese)

Zheng, X., Chen, W., Wang, P., Shen, D., Chen, S., Wang, X., Zhang, Q. and Yang, L. (2016) 'Big data for social transportation', IEEE Transactions on Intelligent Transportation Systems, Vol. 17, No. 3, pp.620-630. 